\title{
DAL LESSICO LATINO AL LESSICO ITALIANO
}

Con questo contributo in onore dello stimato collega Pavao Tekavčić tenteremo un panorama sommario, ma su base sistematica, dei rapporti storici fra il lessico latino e il lessico italiano. Concentrandoci sulla prima tappa di questa storia lessicale considereremo soprattutto alcuni aspetti trattati o accennati anche dal Festeggiato nel terzo volume ("Lessico") della sua magistrale Grammatica storica dell'italiano.

Un problema metodologico fondamentale della storia lessicale è quello dell'oggettività e della rappresentività degli esempi scelti e analizzati. È ovvio che un'adeguata descrizione e caratteristica presuppone una base di materiale se non complessiva almeno di ampiezza rappresentativa. Tale postulato metodologico però è difficile da realizzare, non solo a causa della molteplicità quasi illimitata delle unità lessicali, ma anche per la loro ristretta comparabilità riguardo alla frequenza d'uso. Questo dilemma di principio spiega lo scarso numero di studi sistematici nel campo lessicale - più tipico ancora per la storia dell'italiano ${ }^{1}$ che per altre lingue romanze come il romeno o il francese - e sembra influenzare anche la sistemazione tematica del volume consacrato al 'Lessico' nella Grammatica storica di P. Tekavčić: Nel capitolo introduttivo l'autore dichiara: "L'enorme complessità dei cambiamenti semantici e la nota «resistenza» della semantica alla strutturazione fanno sí che in questa terza parte del manuale di grammatica storica italiana ci concentreremo sulla formazione delle parole e sui prestiti lessicali“ (p. 12). Tuttavia nel capitolo ventesimo intitolato 'Elementi di lessicologia' (p. 167 ss.), dopo la descrizione dettagliata della formazione delle parole e prima di un panorama dei prestiti, il Festeggiato dà pure un'esposizione molto concisa e competente dei principali aspetti della storia lessicale interna, di cui ci occuperemo in seguito.

Per quanto riguarda il postulato suddetto della base obiettiva e rappresentativa, pensiamo che una soluzione metodologica relativamente accettabile sia l'analisi sistematica di un corpus lessicale parziale stabilito sul criterio obiettivo della frequenza. In questo senso esamineremo nel saggio seguente la sorte storica dei mille lessemi latini più frequenti (verbi, sostantivi, aggettivi), quali risultano dal Dictionnaire fréquentiel de la langue latine di L. Delatte (Liège 1981) ${ }^{2}$.

Sullo stato attuale soprattutto della lessicografia cf. M. Pfister, in La dialettologia italiana oggi, Studi offerti a $M$. Cortelazzo, ed. da G. Holtus, M. Metzeltin, M. Pfister, Tübingen 1989, p. 69-79.

2 Si veda anche, con una prospettiva panromanza, il nostro libro Das Schicksal des lateinischen Wortschatzes in den romanischen Sprachen (Passau 1991). 


\section{[La relazione fra latino scritto e latino parlato]}

Riferendosi al lessico del latino classico scritto, il corpus prescelto non riflette certo la base vera e propria delle lingue neolatine, partenti dalle varietà parlate del cosiddetto latino volgare. Comunque, il confronto diacronico sistematico fra il nucleo dei lessemi classici e la situazione linguistica presupposta dagli idiomi romanzi potrà appunto darci un'idea relativamente adeguata delle convergenze o divergenze che si devono presupporre nei rapporti fra il latino scritto e quello parlato (di tarda epoca). I commenti dei linguisti sul carattere generale di quei rapporti sono assai rari, ma ne troviamo uno proprio nel manuale del Tekavčić. Prima di illustrare il dominio "assai importante ed interessante delle differenze fra latino classico e quello parlato", il Festeggiato premette la constatazione seguente: "Che la maggioranza dei vocaboli sia comune ad ambedue (cioè a tutti) i registri di latino, è cosa normale e nota; per l'Italia basti citare PATER, MATER, FILIUS, CANIS, TERRA; DULCIS, SANUS, NOSTER (NOSTRU); ESSERE, HABERE, BIBERE, VIVERE; HODIE, HERI; ET, SI ecc.“ (p. 170). Questa caratteristica, riferita ovviamente al lessico fondamentale (si vedano gli esempi), vale certo per quel campo centrale e viene qui nettamente confermata dai risultati della nostra analisi sistematica: sui mille lessemi più frequenti del latino classico due terzi circa hanno continuatori ereditari nel romanzo, il che ne presuppone l'esistenza anche nel latino parlato o volgare. ${ }^{3}$

Nella quasi metà dei casi però questa esistenza orale sembra limitata - dal punto di vista diatopico e diacronico - , perché soltanto il 37 percento dei lessemi rispettivi sopravvive nella totalità o nella maggioranza delle lingue neolatine. ${ }^{4}-\mathrm{E}$ se teniamo conto anche delle unità lessicali meno frequenti oppure l'insieme del lessico, la quota di continuità romanza, e in linea di massima anche quella di convergenza fra latino scritto e latino parlato, sta diminuendo sensibilmente: il numero totale degli etimi latini delle lingue romanze, che ammonta estensivamente a circa 7 mila lessemi attestati, rappresenta solo una piccola minoranza, cioè tutt'al più il 15 percento di tutti i lessemi latini attestati.

Le differenze lessicali concrete fra il latino scritto e quello parlato o protoromanzo si spiegano nella maggior parte dei casi per certe tendenze generali riassunte nel terzo volume della Storia, p. $170 \mathrm{~s}$., in modo molto chiaro e convincente. - Si può aggiungere che accanto a tali sinonimi preferiti dal linguaggio spontaneo esistono anche divergenze di carattere più fondamentale, in forma di 'significati' scritti classici che nel latino volgare (almeno di tarda epoca) non avevano affatto una corrispondenza lessicale equivalente.

Si tratta da un lato di nozioni espresse nel parlato prevalentemente in forma analitica $o$ in maniera più generale, ad esempio:

3 Fra i 500 lessemi più frequenti, questa percentuale di continuità latino-romanza aumenta a 75 , fra $i$ 100 più frequenti a 90 .

4 Nell'italiano comunque, dialetti inclusi, sono più del 50 percento. 
i valori di verbi tradizionali come CARERE, PATERE, ADIRE, che si rendevano dalle perifrasi analitiche più espressive (e più semplici) NON HABERE, APERTUM ESSE, IRE AD,

le sfumature dei verbi classici di movimento INCEDERE, ABIRE, DISCEDERE, PROFICISCI, PERGERE, rese tutte dal verbo generale per "andare" (cioè IRE oppure VADERE, AMBULARE) ${ }^{5}$, e precisate certo in parte dal contesto situativo della comunicazione orale.

D'altra parte, un certo numero di lessemi classici di carattere astratto o molto specifico, nello sviluppo ereditario verso il romanzo, potevano sparire quasi senza alcun sostituto, perché il loro significato aveva una scarsa importanza nel linguaggio spontaneo e non s'esprimeva quasi mai nelle varietà popolari del latino parlato. Fra gli esempi di questi concetti manifestamente 'poco popolari' troviamo non solo significati schiettamente intellettuali e letterari, ma anche parecchie nozioni come «natura» o «eterno» che a prima vista si direbbero correnti, ma che secondo la testimonianza delle lingue neolatine non avevano una continuità né lessicale né onomasiologica. ${ }^{6}$ - Quando però più tardi le giovani lingue romanze hanno creato un nuovo linguaggio colto e letterario, ebbero bisogno di colmare queste lacune lessicali e ricorrevano spesso al prestito proprio di tali voci latine abbandonate dall'evoluzione popolare.

[La posizione dell'italiano nel processo di frammentazione romanza]

L'individualità del lessico italiano nel dissolversi dell'unità relativa latina e il formarsi di diverse lingue neolatine è stata finora poco analizzata ed è meno conosciuta di quella della maggior parte delle lingue 'sorelle'. Mancando indagini empiriche sistematiche delle realtà concrete, alcuni tentativi di caratterizzazione si basano soprattutto su certe condizioni generali che potevano determinare la differenziazione regionale. Il poco valore spettante a tali deduzioni, a causa innanzi tutto della complessità policausale del fenomeno, risulta per esempio dalla tesi del Gröber che, partendo dal solo principio astratto della cronologia di romanizzazione, giunge alla conclusione che in Italia la lingua protoromanza si sia sviluppata e staccata dal latino più che altrove: "Die am weitesten entwickelte, dem Latein am fernsten gerückte Vulgärsprache lebte danach auf dem heimatlichen Boden Italiens fort, wo sie ihre Gesamtentwicklung durchlief" (in Archiv für lateinische Lexikographie und Grammatik 1, p. 210).

In evidente contrasto con quest'ipotesi, uno sguardo oggettivo sulle realtà linguistiche porta piuttosto a pensare che l'italiano sia una lingua neolatina relativamente conservatrice e rimasta vicina al latino, il che si avvera e conferma infatti se

5 Si vedano le traduzioni rispettive dei lessemi non romanzi nelle Glosse.

6 È ovvio che proprio la sorte ereditaria dei lessemi latini nelle lingue romanze ci offre un criterio metodologico prezioso e relativamente sicuro per la determinazione della loro vitalità orale.

Per quanto riguarda il concetto di "eterno", alcune glosse illustrano che il popolo si accontentava in generale di espressioni approssimative e più concepibili come "per molto tempo» o "per sempre». 
procediamo a un'analisi più sistematica. Quest'analisi contrastiva della maggiore o minore conservatività delle singole lingue romanze può tuttavia considerare o il numero degli 'arcaismi' specifici di ogni regione o il numero complessivo di elementi tradizionali conservati in ciascuna di esse.

In quanto agli arcaismi oppure relitti lessicali sopravvissuti solo in aree isolate, il Festeggiato sottolinea, con pieno diritto, che essi possono per principio caratterizzare ognuna delle lingue romanze: “[...] è un appassionante dominio della lessicologia, in cui ogni idioma neolatino può vantarsi di qualche cimelio importante per la linguistica romanza“ (p. 173 s.). - Quale criterio decisivo per l'attribuzione di un carattere particolarmente conservatore potrà dunque servire solo la loro importanza quantitativa, e da questo punto di vista l'italiano occupa, assieme alla spiccata e nota arcaicità del sardo, il primo posto. Del corpus dei mille lessemi latini più frequenti della lingua classica, due dozzine sopravvivono esclusivamente in italiano o in dialetti italiani, ${ }^{7}$ e precisamente

ADVENTUS $>$ it. ant. avento, AMITTĔRE $>$ ant. amettere, ANIMUS $>$ animo (semidotto, cf. LEI), ATER $>$ ant. adro, CONSPECTUS $>$ cospetto, CONVIVI$\mathrm{UM}>$ ant. combibbia, FIGĔRE $>$ figgere, FLUCTUS $>$ fiotto, IMPIUS > èmpio, NECESSITAS $>$ ant. necistà, PONDUS $>$ pondo, REDIRE $>$ riedere, SOLLICITUS $>$ ant. solecido, TUMULUS $>$ tombolo, inoltre, con spostamento semantico, CLASSIS > chiasso, STUDIUM > ant. stoggio "lusinga, moina, cerimonia“, e, nei dialetti, ACCIPERE (mer.), CARERE (sic.), COGERE (mer.), DUX (ven.), LITUS (ven. $>$ it.), PRAECEPS, SAPIENS (mer. $>$ it.), TEGERE (lucch.). ${ }^{8}$

L'individualità tutto sommato conservatrice dell'italiano nel mondo neolatino risalta in modo ancora più evidente quando effettuiamo l'analisi contrastiva del numero complessivo di lessemi tradizionali conservati nelle singole lingue romanze. I risultati di un tale confronto sono certo da relativizzare di fronte alle divergenze in parte notevoli di ampiezza e di inizio della documentazione e non valgono veramente che per le lingue paragonabili sotto quest'aspetto. Con siffatte riserve risulta però chiaramente che l'italiano conserva una parte molto più grande di ogni altra lingua del patrimonio lessicale latino. Riferendoci al corpus menzionato dei mille lessemi latini più frequenti, otteniamo soltanto nel dominio italiano una quota di continuità ereditaria superiore al 50 percento (cioè 527 unità, o senza i dialetti 447) ${ }^{9}$, e se ci li-

7 Per le parlate sarde il numero rispettivo è inferiore se ci limitiamo agli arcaismi esclusivi, ma leggermente superiore se, per ogni lingua, consideriamo anche le affinità arcaiche con altre aree isolate.

8 Al di fuori del nostro corpus dei lessemi più frequenti vi si aggiungono fra l'altro ACERVUS, CLEMENS, FURIA, FURIOSUS, GREMIUM, MENTUM, MUNDITIA, NEGLEGENS, NEGLEGENTIA, NIMBUS, PECORA e soprattutto la conservazione specificamente italiana e friulana di OMNIS, sottolineata e discussa nella Grammatica storica di P. Tekavčić (II, p. 167 s., III, p. 173). Cf. inoltre B. Migliorini, Storia della lingua italiana, Firenze ${ }^{5} 1978$, p. 20 ss., e M. Cortelazzo, in Lexikon der Romanistischen Linguistik, vol. IV, Tübingen 1988, p. 407.

9 Seguono l'occitanico, il francese e lo spagnolo. 
mitiamo al lessico rispettivo moderno delle singole lingue, giungiamo in ordine di conservatività decrescente ai valori numerici seguenti: ${ }^{10}$

$\begin{array}{cccccccc}\text { italiano } & \text { portoghese } & \text { occitanico } & \text { catalano } & \text { spagnolo } & \text { sardo } & \text { francese } & \text { romeno } \\ 410 & 363 & 351 & 339 & 334 & 300 & 288 & 230\end{array}$

Tuttavia, è ovvio che il lessico italiano non si caratterizza solo per quel tratto generale di relativa conservatività e vicinanza al latino, ma anche per una serie di innovazioni più o meno tipiche dell'evoluzione regionale di questa varietà romanza. Sono in parte innovazioni semantiche come gli sviluppi di CAPERE "prendere" a "capire", CAPTIVUS "prigioniero" a "cattivo", DOMINA "signora" a "donna", IUNGERE "congiungere" a "arrivare, pervenire", SENTIRE "percepire" a "udire", SALIRE "saltare" a "salire", in parte neologismi formali come per esempio i nuovi verbi italiani dimenticare ( $<*$ DEMENTICARE) e scordare ( $<*$ EXCORDARE) di fronte al tipo di denominazione interromanzo *OBLITARE (cf. P. Tekavčić, Grammatica storica, \$1164, G. Rohlfs, Romanische Sprachgeographie, München 1971, §53), o infine influssi stranieri specifici (ad es. longobardi, come schiena). Queste innovazioni specificamente protoitaliane però - benchè non siano ancora state riunite e studiate in modo più sistematico - risultano certo quantitativamente meno importanti di quelle tipiche ad esempio del protofrancese o del protospagnolo, come si può concludere dalle quote rispettive di conservatività sopraindicate. E tale caratteristica della storia individuale dell'italiano viene corroborata inoltre dal fatto che nel quadro del nostro corpus dei mille lessemi latini più frequenti non esiste un solo esempio che manchi unicamente nell'area italiana. ${ }^{11}$

[I latinismi ripresi dal superstrato culturale]

"Si può dire senza esagerare che il latino è presente negli idiomi romanzi, sin dal primissimo inizio della loro vita autonoma, in due filoni, o strati: quello autoctono e popolare, e l'altro, dovuto ai contatti tra il latino scritto, medievale, e le giovani lingue neolatine. [...] Da quando esistono, gli idiomi neolatini occidentali attingono al latino - e tramite il latino, al greco - ogniqualvolta hanno bisogno di un nuovo termine, religioso, filosofico, artistico, scientifico ecc. " (Tekavčić, Grammatica storica, III, p. 174). ${ }^{12}$ - Esaminando gli esempi concreti di questi prestiti dotti, osserviamo che tramite il latino medievale si riprendono anche una serie di voci del lessico tradizionale classico e che oltre ai nuovi termini specifici si tratta pure di lessemi di

10 Anche W. Mańczak, su base dell'analisi comparativa di un testo biblico, giunge al risultato che “quand on tient compte de la totalité des faits, c'est-à-dire des textes, il est indubitable que c'est l'italien qui est la langue la plus archaïque" (in Actes du XVIT Congrès International de Linguistique et Philologie Romanes, Aix-en-Provence 1985, II, p. 111-130), e M. Cortelazzo, l. cit., parla di una "eredità massiccia [...] che rende l'italiano molto più vicino alla matrice latina delle altre lingue romanze" (p. 401).

11 Mentre per esempio il francese ignora continuatori di DARE, INCENDERE, MENS, SOL, lo spagnolo di ARGENTUM, CANIS, NEPOS, VELLE e il romeno si caratterizza per un numero particolarmente alto di tali lacune specifiche (cf. ad es. C. Th. Gossen, in Vox Romanica 41, 1982, p. 13-45).

12 Cf. fra l'altro anche C. Tagliavini, Le origini delle lingue neolatine, Bologna ${ }^{6} 1972$, $\$ 61$ 'Il superstrato culturale latino'. 
carattere più generale come i già menzionati NATURA o AETERNUS “eterno“" (mutuati da quasi tutte le lingue romanze, ad es. dall'italiano fin dal Duecento).

Sono dunque in gran parte parole che il latino parlato (almeno di tarda epoca) aveva abbandonato come più o meno dispensabili (si veda sopra), ma che per le nuove esigenze di una comunicazione colta e scritta, tenuta all'espressione più differenziata, precisa ed esplicita, si rivelarono di nuovo utili se non necessarie. ${ }^{13}$ In tal modo - per farci un'idea dell'importanza quantitativa del fenomeno - fra i lessemi latini del nostro corpus che, sul filone popolare dell'evoluzione ereditaria, non sono sopravvissuti affatto o appena, due terzi circa sono stati ripresi per via colta da una o parecchie delle lingue romanze. $E$ benchè all'inizio appartenessero soprattutto a certi ambienti specifici (ad es. natura al linguaggio filosofico o eterno al linguaggio religioso), i latinismi potevano spesso estendersi, secondariamente, al lessico comune delle lingue moderne. Così, fra le voci storicamente 'dotte', nel quadro del nostro corpus, più di cinquanta figurano oggi nel lessico fondamentale delle mille parole più frequenti dell'italiano ${ }^{14}$ (ad es. animale, caso, causa, concedere, condizione, difficile, dividere, facile, felice, genere, immagine, immenso, libero, modo, natura, necessario, occasione, occupare, permettere, preferire, principio, ripetere, silenzio, simile, spirito, utile).

Dal punto di vista comparativo, l'italiano, a confronto con le altre lingue neolatine, si caratterizza per un numero particolarmente alto di latinismi e perciò per una vicinanza, anche su quest'aspetto dotto, relativamente stretta al lessico latino. L'Italia, che in quanto alle condizioni esteriori era, secondo C. Tagliavini, "il paese che si trovava più esposto a subire l'influsso del superstrato culturale latino" ( $l$. cit.), attingeva difatti ben più parole al latino che ogni altra lingua: riferendoci di nuovo all'analisi del nostro corpus, fra i circa 630 dei lessemi latini che sono più o meno abbandonati ereditariamente, l'italiano mutua in totale 390 forme (il francese 296, lo spagnolo 323 , il portoghese 342 ) e se ci si limita alle parole tuttora viventi, la quota per l'italiano rimane più del 50 percento (324), mentre nel francese moderno si riduce a un terzo (200) e nello spagnolo al 45 percento circa (292). Tre dozzine circa delle parole latine rispettive vengono riprese unicamente o quasi solo dall'italiano, fra le quali, per dare qualche esempio, DESIDERIUM (desiderio), EXPERIRI (esperire), FAX (face lett.), MITIS (mite), REPERIRE (reperire), RUPES (rupe). ${ }^{15}$

13 D'altra parte, il movimento latineggiante si manifesta anche nella sostituzione di denominazioni tradizionali da nuovi sinonimi dotti (ad es. esercito in vece di oste, cf. F. Maggini, in Lingua Nostra 3, 1941 , p. 76-97) o persino da varianti rilatinizzate della stessa parola (ad es. invidia in vece di inveggia, superare in vece di soprare, virtù in vece di vertù).

14 Si veda A. Juilland / V. Traversa, Frequency Dictionary of Italian Words, Den Haag - Paris 1973.

\$5 Il numero totale di corrispondenza sia ereditaria sia dotta col lessico latino più frequente del nostro corpus ammonta per l'italiano moderno a circa 730 (sia 410 più 324) e si allarga estensivamente, se includiamo i dialetti e la vecchia lingua, a più di 900 (su 1000). 


\section{[Il lessico latino classico e la lingua di Dante]}

Sintetizzando i rapporti sia ereditari sia dotti fra il latino e la tappa dell'italiano antico, consideriamo infine i dati quantitativi quali risultano dal lessico individuale concreto della lingua di Dante. L'ampiezza e la diversità del vocabolario dantesco ${ }^{16}$ riflette certo una larga parte del lessico italiano tradizionale della sua epoca e illustra d'altronde in che misura l'arricchimento dotto per mezzo dei latinismi lessicali si faceva già valere all'inizio del Trecento.

Prendendo per base il corpus dei mille lessemi latini più frequenti, notiamo che Dante valorizza nei suoi testi italiani pressappoco l'intero patrimonio lessicale ereditario, vale a dire quasi 420 lessemi, il che corrisponde a più del 90 percento del totale delle unità sopravvissute in italiano. Il 5 percento al massimo di quei lessemi tradizionali utilizzati dal 'padre della lingua' spariscono nel corso dell'evoluzione posteriore e sono sconosciuti dall'italiano moderno (ad es. donno "signore", ducere "condurre", lecere "essere lecito", manere "rimanere", oste "esercito", soprare "superare", spazzo "suolo", suora "sorella"). ${ }^{17}$

Per quanto riguarda i latinismi, pressappoco due terzi di tutte le forme mutuate dall 'italiano al superstrato culturale (cioè 240 circa di 390$)^{\mathbf{1 8}}$ si trovano già in Dante, e la stragrande maggioranza di loro si è durevolmente integrata nella lingua. ${ }^{19} \mathrm{Ma}$ l'importanza fondamentale di Dante per la costituzione del lessico dotto italiano appare soprattutto nel fatto che per la quasi metà di quei latinismi la sua lingua ci offre la prima attestazione italiana. ${ }^{20}$ Citiamo fra queste voci dotte attestate propriamente a partire da Dante (in totale circa 110 nel quadro del nostro corpus): accedere, astro, cedere, differire, egregio, esperire, felice, frequente, genere, immagine, immenso, ingrato, inizio, libertà, mesto, mite, noto, ottenere, ufficio, ozio, perpetuo, potenza, principe, principio, pudore, regione, ripetere, resistere, rupe, simile, spazio, tacito, tenebre, tollerare, utile, vittoria, voluttà. ${ }^{21}$ E nella prospettiva compara-

16 Si veda soprattutto la Enciclopedia dantesca, ed. da U. Bosco, Roma 1970-1976.

17 Più numerosi sono i lessemi appartenenti oggi al solo vocabolario letterario come fiotto, mirare, riedere.

18 Fra i latinismi ancora assenti nel lessico di Dante menzioniamo fra l'altro ambizione, assiduo, avido, esito, difficile, facile, ignorare, mole, occasione, omettere, premio, supplizio, terrore, valido, vasto.

19 Come esempi di prestiti dotti isolati che non riuscirono a imporsi si confrontino fra l'altro crebro (CREBER), cupere (CUPERE), curro (CURRUS), igne (IGNIS), ludere (LUDERE), muno (MUNUS), relinquere (RELINQUERE), ruere (RUERE), tuto (TUTUS), vincire (VINCIRE), viro (VIR).

20 Si vedano per la datazione soprattutto $\mathrm{M}$. Cortelazzo / P. Zolli, Dizionario etimologico della lingua italiana (DELI), Bologna 1979-1988, e G. Colussi (ed.), Glossario degli antichi volgari italiani (GAVI), Helsinki 1983 ss.

Una gran parte dei latinismi anteriori a Dante si trovano per la prima volta in Brunetto Latini. Parecchie forme attestate anteriormente non diventano correnti che nella lingua di Dante (ad es. onesto, cf. DELI).

21 S'intende che il valore stilistico e talvolta anche semantico dei latinismi nell'uso di Dante poteva divergere dall'uso moderno. Così, rispetto al valore per esempio di egregio B. Migliorini (cit. in DELI) precisa che aveva nel linguaggio "dantesco un tono molto più alto, secondo l'etimologia, che non sia quello odierno", e riguardo a mesto I. Baldelli (in Enciclopedia dantesca) scrive: "Evidentemente la parola, che oggi in italiano ha un valore piuttosto tenue, o almeno non particolarmente drammatico, è sentita da Dante col valore originale latino, cioè «disperato», «tristissimo»". 
tiva romanza colpisce che molti di questi lessemi latini ripresi da Dante (o prima) vengono mutuati nelle altre lingue neolatine nettamente più tardi (ad es. CEDERE, INGRATUS, PUDOR, TOLERARE, VOLUPTAS) o che persino non hanno affatto 0 appena corrispondenze dotte al di fuori dell'italiano (ad es. i già citati EXPERIRI, FAX, MITIS, REPERIRE, RUPES).

Se, in conclusione, riassumiamo l'affinità complessiva del lessico italiano di Dante con quello del latino classico, risulta dunque, nel quadro delle mille unità latine più frequenti, una quota di corrispondenza di due terzi (circa 420 esempi di continuità popolare e 240 di ripresa dotta), il che rappresenta in confronto alla situazione nelle altre lingue neolatine e dei loro autori o testi rispettivi una vicinanza particolarmente stretta e distintiva.

Povzetek

\section{OD LATINSKEGA BESEDNEGA ZAKLADA K ITALIJANSKEMU}

Prispevek analizira prve etape zgodovine italijanskega besedja, in sicer na podlagi opazovanja usode tisoč najbolj pogostih latinskih leksemov. Primerjava med osrednjim leksikalnim korpusom klasične latinšcine in predpostavljenim jezikovnim stanjem $\mathrm{v}$ romanskih jezikih dovoljuje razmeroma pravilno gledanje na odnos, v besedišču, med pisano in (v pozni dobi) govorjeno latinščino. Od teh tisoč najbolj pogostih leksemov se jih približno dve tretjini nadaljuje v romanskih jezikih, kar naj bi pótrjevalo njihovo eksistenco v vulgarni latinščini. Od celotnega besednega zaklada se v romanskih jezikih ohranja samo 15 odstotkov. Tako lahko za govorjeno latinščino zagotovimo izbiro ekspresivnejših sinonimov, pa tudi bolj globinska razhajanja. Prenekateri 'pomen' v spontanem govoru ni imel ustreznega izraza: govorjena beseda se je zatekla k opisni obliki, ali k splošnejšemu izrazu, ali pa je izraz celo brez škode opustila, kadar je pač šlo za izrazito abstraktne pojme. Individualnost italijanskega besedišča se $\mathrm{v}$ romanski razdrobljenosti kaže kot posebej konservativna. Ohranja prav toliko arhaizmov kot sardščna, v celoti pa ima večje število podedovanih besed od drugih romanskih jezikov; med tisoč najpogostejšimi leksemi jih gre v italijanščini več kot polovica med iz latinščine podedovane besede. Tako je italijanščina latinšcini razmeroma blizu; ta vtis bližine, podobnosti, bi rekli, se še poveča zaradi dotoka kulturnega superstrata, ki je bil v italijanščini zmeraj močnejši kot drugod: razčlenjeni korpus kaže, da je italijanščina šla na pósodo $v$ opuščeno latinsko besedišč - to je ravno značilnost t.i. učenih besed - za 390 izrazov, in od tega so trije ducati teh latinizmov znani samo iz italijanščine. Vezanost italijanskega besedišča na latinsko, in to je lahko razlikovalni kriterij do drugih romanskih jezikov, se zelo jasno vidi tudi v obsežnem Dantejevem besednem zakladu. Pesnik je uporabil več kot $90 \%$ podedovanega latinskega fonda, rabi pa tudi dve tretjini vseh latinizmov, kar jih je italijanščina prevzela; in prav pri njem je prvič izpričana skoraj polovica teh učenih, neljudskih oblik. 